\title{
O impacto da pandemia de COVID-19 nos transtornos alimentares e seu tratamento: uma revisão integrativa da literatura
}

The impact of the COVID-19 pandemic on eating disorders and their treatment: an integrative literature review

El impacto de la pandemia de COVID-19 en los trastornos alimentarios y su tratamiento: una revisión integradora de la literatura

Carolina de Oliveira Coutinho ORCID: https://orcid.org/0000-0001-7517-6543 Universidade do Estado do Rio de Janeiro, Brasil E-mail: carolocoutinho@hotmail.com

Thamires Monteiro Laranjeira Mota ORCID: https://orcid.org/0000-0002-9762-6282 Universidade do Estado do Rio de Janeiro, Brasil E-mail: thamiresmonteiro@gmail.com

Larissa Pereira Santos ORCID: https://orcid.org/0000-0002-9419-0946 Universidade do Estado do Rio de Janeiro, Brasil E-mail: santoslarissap@gmail.com

Thainá Santana da Silva ORCID: https://orcid.org/0000-0003-0546-2661 Universidade do Estado do Rio de Janeiro, Brasil E-mail: santanathainas1@gmail.com

Thais Nascimento Conde ORCID: https://orcid.org/0000-0001-9926-1662 Universidade do Estado do Rio de Janeiro, Brasil E-mail: thaisnconde@gmail.com

Alessandra da Rocha Pinheiro Mulder ORCID: https://orcid.org/0000-0001-5406-7903 Universidade do Estado do Rio de Janeiro, Brasil E-mail: alessandra.mulder@gmail.com

Cristiane Marques Seixas ORCID: https://orcid.org/0000-0003-2630-9746 Universidade do Estado do Rio de Janeiro, Brasil E-mail: cris.marques.seixas@gmail.com

\begin{abstract}
Resumo
O contexto da pandemia provocada pela COVID-19 tem gerado alterações profundas em nossas condições de vida e no modo como nos relacionamos, apresentando potencial expressivo de impacto na saúde mental, especialmente entre indivíduos com transtornos mentais pré-existentes, dentre os quais se destacam os transtornos alimentares. Deste modo, objetivou-se investigar como a pandemia por COVID-19 pode impactar no desenvolvimento, manutenção e tratamento de quadros de transtornos alimentares. Adotando-se abordagem qualitativa e perspectiva descritiva, foi realizada revisão integrativa da literatura por meio de busca às bases de dados LILACS e PUBMED e biblioteca eletrônica SciELO. Incluíram-se estudos de intervenção publicados a partir de 2020 e que relacionavam os quadros de transtornos alimentares à pandemia por COVID-19. Foram selecionados 14 estudos, analisados a partir da categorização em eixos temáticos. Os resultados apontam que os fatores situacionais associados à pandemia de COVID-19 podem influenciar de maneira significativa na precipitação dos quadros de transtornos alimentares, assim como no agravamento da psicopatologia geral e específica, apesar da grande variabilidade identificada entre os estudos. Além disso, podem exacerbar as barreiras de acesso aos cuidados em saúde. Os dados apresentados podem ser úteis na construção de caminhos possíveis para sustentar o tratamento de indivíduos com transtornos alimentares e evidenciam o tamanho do desafio enfrentado para levar a cabo os tratamentos que associam a gravidade dos transtornos alimentares a uma realidade que leva cotidianamente à deterioração da saúde mental dos brasileiros.
\end{abstract}

Palavras-chave: Transtornos da alimentação e da ingestão de alimentos; Saúde mental; COVID-19; Coronavírus. 


\begin{abstract}
The context of the pandemic caused by COVID-19 has generated profound changes in our living conditions and the way we interact, with an expressive potential impact on mental health, especially individuals with pre-existing mental disorders, including eating disorders. Thus, the aim was to investigate how the COVID-19 pandemic can impact the development, maintenance and treatment of eating disorders. Adopting a qualitative approach and a descriptive perspective, an integrative literature review was carried out by searching the LILACS, PUBMED and the SciELO electronic library databases. Intervention studies published since 2020 onwards and which related eating disorders to the COVID-19 pandemic were included. Fourteen studies were selected, analyzing the categorization in thematic axes. The results show that situational factors associated with the COVID-19 pandemic can significantly influence the precipitation of eating disorders, as well as the worsening of general and specific psychopathology, despite the great variability identified between studies. Furthermore, they can exacerbate barriers to accessing health care. The data presented can be useful in building possible ways to support the treatment of individuals with eating disorders and show the size of the challenge faced in carrying out treatments that associate the severity of eating disorders with a reality that leads to the deterioration of mental health of Brazilians on a daily basis.
\end{abstract}

Keywords: Eating and feeding disorders; Mental health; COVID-19; Coronavirus.

\title{
Resumen
}

El contexto de la pandemia provocada por el COVID-19 ha generado cambios profundos en nuestras condiciones de vida y en la forma en que interactuamos, con un potencial expresivo de impacto en la salud mental, especialmente entre las personas con trastornos mentales preexistentes, entre los que se encuentran los trastornos alimentarios. Por lo tanto, el objetivo fue investigar cómo la pandemia de COVID-19 puede afectar el desarrollo, mantenimiento y tratamiento de los trastornos alimentarios. Adoptando un enfoque cualitativo y una perspectiva descriptiva, se realizó una revisión integradora de la literatura mediante la búsqueda en las bases de datos LILACS y PUBMED y en la biblioteca electrónica SciELO. Se incluyeron los estudios de intervención publicados a partir de 2020 y que relacionaron los trastornos alimentarios con la pandemia de COVID-19. Se seleccionaron catorce estudios, analizados a partir de la categorización en ejes temáticos. Los resultados muestran que los factores situacionales asociados a la pandemia de COVID-19 pueden influir significativamente en la precipitación de los trastornos alimentarios, así como en el agravamiento de la psicopatología general y específica, a pesar de la gran variabilidad identificada entre estudios. Además, pueden exacerbar las barreras para acceder a la atención médica. Los datos presentados pueden ser útiles para construir posibles formas de apoyar el tratamiento de las personas con trastornos alimentarios y mostrar la magnitud del desafío al que se enfrenta la realización de tratamientos que asocian la gravedad de los trastornos alimentarios con una realidad que a diario conduce al deterioro de la salud mental de los brasileños.

Palabras clave: Trastornos de la conducta alimentaria y de la ingesta de alimentos; Salud mental; COVID-19; Coronavirus.

\section{Introdução}

Uma grave crise sanitária global tem sido enfrentada com a emergência do SARS-CoV-2, vírus com alta taxa de transmissibilidade e responsável pela galopante proliferação da COVID-19 em todo mundo. Desde que a Organização Mundial de Saúde (OMS) decretou Emergência de Saúde Pública de Importância Internacional, em 30 de janeiro de 2020 (World Health Organization, 2020d), diversos países vêm desenvolvendo pesquisas para avaliar o impacto da pandemia sobre populações específicas. Atualmente, a doença é tida como a maior emergência de saúde pública enfrentada pela comunidade internacional em décadas, com repercussões importantes nos domínios político, econômico e psicossocial o que requer, portanto, um trabalho em escala nacional e mundial a fim de frear seu o ritmo de expansão (World Health Organization, 2020b).

Trata-se de uma pandemia com características ímpares, dada a extensão, velocidade de crescimento e impacto geral na população e nos serviços de saúde. Além disso, apesar de atualmente já conhecermos os riscos clínicos, transmissibilidade, infectividade, letalidade e mortalidade, as sequelas a médio e longo prazo ainda são pouco conhecidas.

As projeções iniciais de demandas avassaladoras de recursos de saúde e subsequentes mortes relacionadas à doença levaram à adoção de medidas de saúde pública com o intuito de reduzir a rápida propagação da transmissão interpessoal, que se dá basicamente pelo contato direto com pessoa infectada, com objetos e superfícies contaminadas e por meio da inalação de aerossóis contendo o vírus (Bourouiba, 2020; Morawska \& Cao, 2020; World Health Organization, 2020b). Deste modo, 
inicialmente, considerando-se a ausência de imunidade da população e a inexistência de uma vacina e tratamentos medicamentosos eficazes, a OMS, apoiando-se em experiências anteriores de controle de neo epidemias virais, orientou a adoção de medidas não farmacológicas para a contenção da pandemia de COVID-19. Entre tais medidas estão o distanciamento físico (em várias dimensões chegando até o lockdown completo), medidas de controle sanitário - como a imposição de quarentena aos casos suspeitos e confirmados de COVID-19 e seus respectivos contatos - e a recomendação do uso de máscaras e higienização correta e frequente das mãos (World Health Organization, 2020a, 2020c).

Cabe destacar que tais medidas foram e ainda têm sido implementadas de modos distintos nos diferentes países e seus resultados, possivelmente, dependem não apenas dos mecanismos operacionais utilizados na implementação, como também de aspectos socioeconômicos, culturais e de características dos sistemas políticos e de saúde. Ressalta-se que no cenário global, até a primeira semana de junho de 2021 o Brasil é o segundo país com maior número de óbitos por COVID-19, atrás apenas dos Estados Unidos, segundo dados da OMS (World Health Organization, 2021).

Esse preocupante contexto da pandemia provocada pela COVID-19, tem gerado alterações profundas em nossas condições de vida e no modo como nos relacionamos. O medo que se apresenta com suas mais variadas faces e intensidades, assim como as inseguranças de toda ordem que emergiram com a pandemia, junto com as perdas de familiares e pessoas queridas entre outros fatores contribuem para que um contingente significativo da população apresente níveis bastante elevados de ansiedade, estresse, sentimentos de desamparo e solidão, preocupação e sensação de falta de controle diante das inúmeras incertezas do momento. Assim sendo, há um potencial expressivo de incremento de agravos à saúde mental e intensificação dos sintomas em indivíduos com transtornos mentais pré-existentes (Fiorillo \& Gorwood, 2020; Torales et al., 2020; Wang et al., 2020).

Notavelmente, alguns grupos podem ser mais vulneráveis aos efeitos da pandemia atual na saúde mental, sendo desproporcionalmente impactados, dentre os quais se destacam os transtornos alimentares (Pfefferbaum \& North, 2020; Reger et al., 2020). Esses quadros podem ser definidos, a partir da literatura biomédica, como um conjunto de síndromes clínicas caracterizadas por graves alterações do comportamento alimentar, por sofrimento e/ou preocupação excessiva com a forma ou o peso corporal (American Psychiatric Association, 2013).

Também é importante ressaltar que a assistência a indivíduos com transtornos alimentares está sendo afetada pela pandemia como consequência da necessidade de se impor restrições para que se cumpra o distanciamento físico - o que pode servir como uma razão para evitar serviços de saúde mental -, pelo risco potencialmente maior de se contrair COVID-19 por estar fisicamente presente em uma unidade de saúde e, em alguns casos, pelo esgotamento dos recursos de saúde.

Por outro lado, pode-se identificar no contexto de pandemia um potencial de mitigação dos gatilhos sociais interpessoais relacionados à comportamentos alimentares tidos como desordenados e insatisfação corporal como consequência, por exemplo, da redução de oportunidades para comparações sociais baseadas no corpo (Cooper et al., 2020; Saunders et al., 2019).

Diante do exposto, o objetivo deste trabalho foi descrever como a pandemia por COVID-19 pode impactar no desenvolvimento, manutenção e tratamento de quadros de transtornos alimentares, analisando o panorama das publicações sobre os transtornos alimentares durante a pandemia de COVID-19 em periódicos científicos nacionais e internacionais.

\section{Metodologia}

Trata-se de uma pesquisa de natureza qualitativa, baseada nos pressupostos da revisão integrativa de literatura. A escolha por tal metodologia se justifica pelo seu potencial de proporcionar a síntese do conhecimento atual sobre uma temática específica - a partir da identificação, análise e síntese de resultados de estudos independentes sobre um mesmo tema - 
impactando no desenvolvimento de pensamento crítico e contribuindo, deste modo, com uma possível repercussão positiva na qualidade dos cuidados prestados em saúde (Souza et al., 2010).

Para localização dos estudos científicos correspondentes aos objetivos da pesquisa realizou-se uma revisão de literatura nas bases de dados LILACS e PUBMED e na biblioteca eletrônica SciELO em fevereiro de 2021, utilizando-se, dois conjuntos de termos de busca, selecionados de acordo com os Descritores em Ciências da Saúde (DeCS) e Medical Subject Headings (MeSH). Foram combinados e cruzados individualmente: "eating disorders", "anorexia nervosa", "bulimia nervosa", "binge eating disorder"; "COVID-19", "coronavirus" "pandemic". Além disso, com o intuito de ampliar o campo empírico a ser analisado, realizou-se uma pesquisa complementar nas referências dos artigos selecionados a fim de localizar possíveis estudos não identificados nas buscas.

Foram incluídas pesquisas publicadas a partir de 2020, ano em que foi decretada a pandemia pelo novo coronavírus. Os artigos repetidos foram excluídos e as publicações foram pré-selecionadas pelos seus títulos, os quais deveriam conter o termo completo e/ou referências aos quadros de transtornos alimentares em correlação com a pandemia de COVID-19. Em seguida, foi realizada a leitura dos resumos dos artigos para identificação e posterior inclusão daqueles que se tratavam de estudos de intervenção. Nos casos de incerteza, o texto completo foi consultado para confirmar a elegibilidade do estudo. Não foram incluídos artigos de revisão, editoriais, comentários, fóruns clínicos ou manuscritos teóricos (Figura 1).

Dois autores independentes realizaram as buscas nas bases de dados e a avaliação dos títulos e resumos dos artigos selecionados, em conformidade com os critérios de inclusão/exclusão pré-definidos, para que fossem eleitos aqueles que deveriam ser lidos na íntegra. Não houve divergências entre os revisores sobre a inclusão dos manuscritos. 
Figura 1. Fluxograma do processo de seleção das publicações que constituíram a amostra.

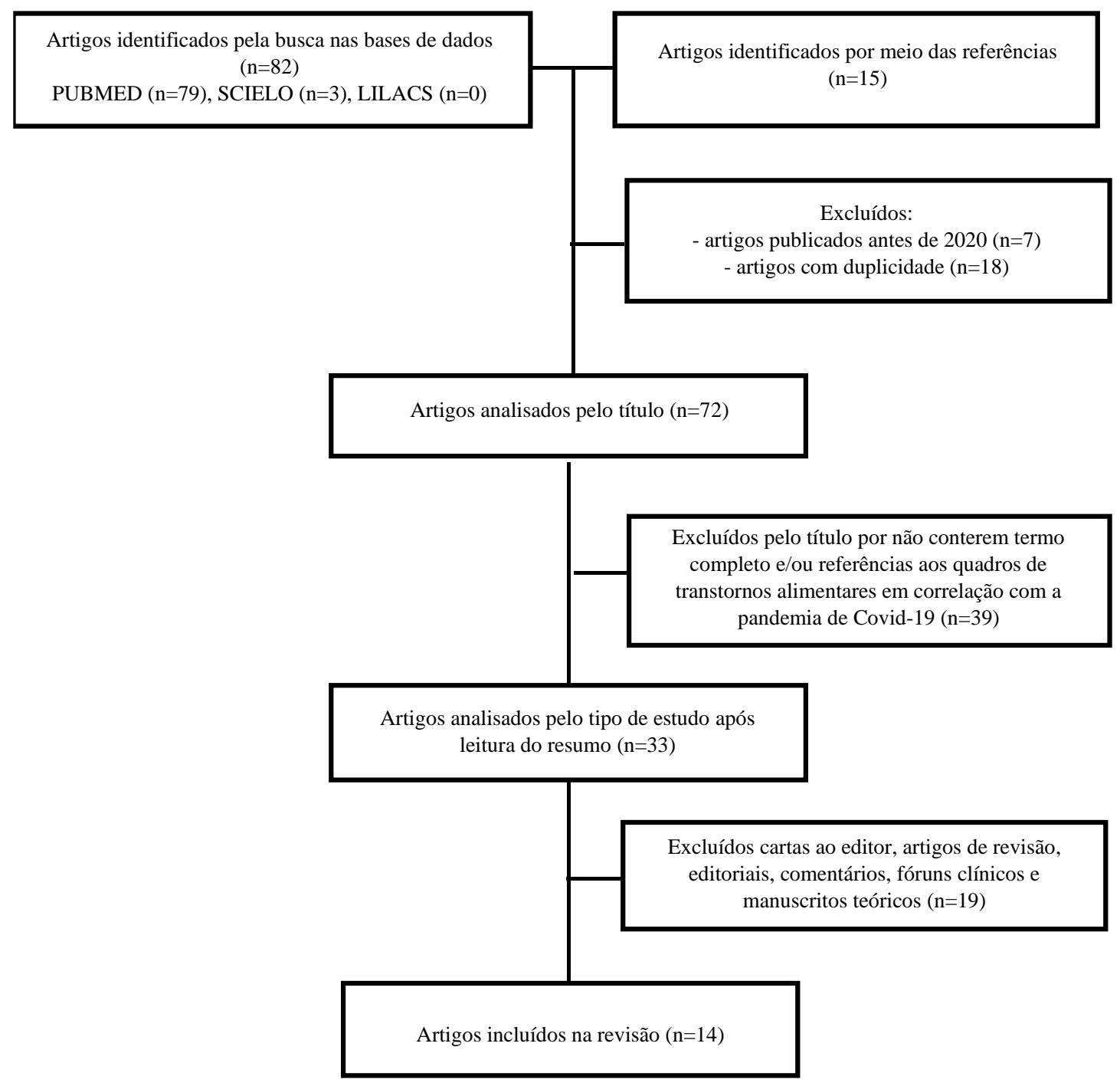

Fonte: Autores.

Como sinalizado na Figura 1, a busca resultou em 97 artigos localizados, sendo 79 no PUBMED, 3 na LILACS, nenhum na SciELO e 15 identificados por meio da busca complementar às referências dos artigos selecionados. Destes, foram descartados 7 artigos publicados antes de 2020 e 18 presentes em duplicidade na amostra. Totalizou-se então 72 artigos, dos quais 39 foram excluídos após análises de seus títulos e 33 por não se tratarem de estudos de intervenção. Deste modo, ao final foram selecionados 14 artigos para compor esta revisão, tendo sido lidos na íntegra. Cabe destacar que não foram encontrados artigos publicados no Brasil.

\section{Resultados}

Todos os artigos selecionados referem-se a estudos realizados durante a pandemia de COVID-19. Pode-se identificar diferenças entre estudos realizados nas primeiras semanas de eclosão da pandemia e estudos mais tardios que podem recuperar efeitos posteriores das adaptações de protocolos de atendimento e do agravamento ou melhora dos sintomas relacionados aos transtornos alimentares. Por se tratar de uma pandemia de proporções globais, esses efeitos foram se dando em momentos 
diferentes e se relacionaram com as medidas adotadas em cada país para o enfrentamento da crise. Nesse sentido, os estudos analisados distribuem-se segundo eixos temáticos que são apresentados no Quadro 1.

Quadro 1. Caracterização das publicações e divisão em eixos temáticos

\begin{tabular}{|c|c|c|c|c|}
\hline & Autores/Ano & Título & Objetivo & Principais achados \\
\hline 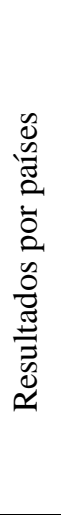 & $\begin{array}{l}\text { Phillipou et al. } \\
\text { (2020) }\end{array}$ & $\begin{array}{l}\text { Eating and exercise } \\
\text { behaviors in eating } \\
\text { disorders and the } \\
\text { general population } \\
\text { during the COVID-19 } \\
\text { pandemic in Australia: } \\
\text { Initial results from the } \\
\text { COLLATE project. }\end{array}$ & $\begin{array}{l}\text { Identificar e caracterizar as } \\
\text { mudanças no } \\
\text { comportamento alimentar e } \\
\text { prática de exercícios físicos } \\
\text { em uma amostra } \\
\text { australiana de indivíduos } \\
\text { com transtornos } \\
\text { alimentares e na população } \\
\text { em geral. }\end{array}$ & $\begin{array}{l}\text { Os resultados apontam que entre indivíduos com } \\
\text { transtornos alimentares, a maior parte } \\
\text { apresentava níveis moderados a extremamente } \\
\text { graves de depressão, ansiedade e estresse. Além } \\
\text { disso, identificou-se aumento da restrição } \\
\text { alimentar com o objetivo de influenciar o peso ou } \\
\text { forma corporal, compulsão alimentar, purgação e } \\
\text { da prática de exercícios. Na população em geral, } \\
\text { a maioria dos entrevistados relatou "níveis } \\
\text { normais" de ansiedade, depressão e estresse, } \\
\text { comportamentos restritivos e de compulsão } \\
\text { alimentar, bem como diminuição da prática de } \\
\text { exercícios físicos em relação ao período anterior } \\
\text { à pandemia. }\end{array}$ \\
\hline 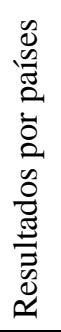 & $\begin{array}{c}\text { Branley-Bell \& } \\
\text { Talbot. (2020) }\end{array}$ & $\begin{array}{l}\text { Exploring the impact } \\
\text { of the COVID-19 } \\
\text { pandemic and UK } \\
\text { lockdown on } \\
\text { individuals with } \\
\text { experience of eating } \\
\text { disorders. }\end{array}$ & $\begin{array}{l}\text { Investigar o impacto da } \\
\text { pandemia de COVID-19 e } \\
\text { do lockdown na vida de } \\
\text { pessoas com experiências } \\
\text { de transtornos alimentares } \\
\text { residentes no Reino Unido. }\end{array}$ & $\begin{array}{l}\text { Os resultados sugerem impactos prejudiciais no } \\
\text { bem-estar psicológico dos indivíduos } \\
\text { pesquisados. O estudo aponta a necessidade de } \\
\text { intervenções de apoio a esta população durante e } \\
\text { após a pandemia. As intervenções devem ser co- } \\
\text { projetadas com os usuários para favorecer a } \\
\text { eficácia do tratamento. Os resultados também } \\
\text { destacam as desigualdades na oferta de serviços } \\
\text { de saúde. }\end{array}$ \\
\hline 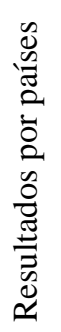 & $\begin{array}{l}\text { Brown et al. } \\
\quad(2021)\end{array}$ & $\begin{array}{c}\text { A qualitative } \\
\text { exploration of the } \\
\text { impact of COVID-19 } \\
\text { on individuals with } \\
\text { eating disorders in the } \\
\text { UK. }\end{array}$ & $\begin{array}{l}\text { Explorar o impacto do } \\
\text { COVID-19 e as medidas de } \\
\text { saúde pública associadas } \\
\text { em adultos com transtornos } \\
\text { alimentares no Reino } \\
\text { Unido. }\end{array}$ & $\begin{array}{l}\text { O impacto do lockdown relacionado à pandemia } \\
\text { de COVID-19 foi considerado tanto um } \\
\text { catalisador para comportamentos alimentares } \\
\text { desordenados como incentivo à recuperação. O } \\
\text { estudo aponta a importância de profissionais dos } \\
\text { serviços de apoio adaptarem suas estratégias de } \\
\text { tratamento para ajudar indivíduos com } \\
\text { transtornos alimentares a lidarem com as } \\
\text { dificuldades que surgiram com a pandemia. }\end{array}$ \\
\hline 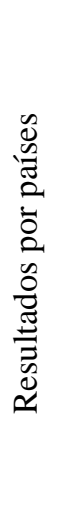 & $\begin{array}{l}\text { Termorshuizen } \\
\text { et al. (2020) }\end{array}$ & $\begin{array}{l}\text { Early impact of } \\
\text { COVID- } 19 \text { on } \\
\text { individuals with self- } \\
\text { reported eating } \\
\text { disorders: A survey of } \\
\sim 1,000 \text { individuals in } \\
\text { the United States and } \\
\text { the Netherlands. }\end{array}$ & $\begin{array}{l}\text { Avaliar o impacto inicial } \\
\text { da COVID-19 em pessoas } \\
\text { com transtorno alimentar } \\
\text { autorrelatado. }\end{array}$ & $\begin{array}{l}\text { Os resultados revelaram efeitos importantes sobre } \\
\text { os sintomas inerentes aos quadros de transtornos } \\
\text { alimentares, com o aumento da restrição } \\
\text { alimentar e medo de não ser capaz de encontrar } \\
\text { alimentos compatíveis com seu plano alimentar. } \\
\text { Também foi relatado o aumento da frequência de } \\
\text { episódios de compulsão alimentar, no desejo por } \\
\text { comer compulsivamente e aumento nos níveis de } \\
\text { ansiedade e maiores preocupações sobre o } \\
\text { impacto da COVID-19 na saúde mental do que } \\
\text { na saúde física. Entretanto, os entrevistados } \\
\text { também notaram efeitos positivos relacionados à } \\
\text { relação com a família, ao autocuidado e à } \\
\text { motivação para recuperação. }\end{array}$ \\
\hline 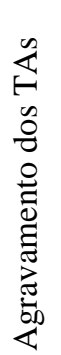 & $\begin{array}{l}\text { Machado et al. } \\
\text { (2020) }\end{array}$ & $\begin{array}{l}\text { Impact of COVID-19 } \\
\text { lockdown measures on } \\
\text { a cohort of eating } \\
\text { disorders patients. }\end{array}$ & $\begin{array}{l}\text { Avaliar o impacto das } \\
\text { medidas de bloqueio em } \\
\text { uma coorte de pacientes } \\
\text { com transtornos } \\
\text { alimentares acompanhados } \\
\text { como parte de um estudo } \\
\text { de tratamento naturalístico } \\
\text { em andamento. }\end{array}$ & $\begin{array}{l}\text { Os resultados sugerem que o impacto do } \\
\text { lockdown foi significativamente correlacionado } \\
\text { com os sintomas de transtornos alimentares e } \\
\text { associados ao aumento da impulsividade, } \\
\text { dificuldades na regulação da emoção e prejuízo } \\
\text { clínico pós-lockdown. Os achados evidenciam a } \\
\text { necessidade de estratégias de intervenção para } \\
\text { mitigar o impacto negativo da pandemia sobre a } \\
\text { psicopatologia dos transtornos alimentares. }\end{array}$ \\
\hline
\end{tabular}




\begin{tabular}{|c|c|c|c|c|}
\hline 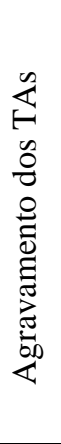 & $\begin{array}{l}\text { Castellini et al. } \\
\qquad(2020)\end{array}$ & $\begin{array}{l}\text { The impact of COVID- } \\
19 \text { epidemic on eating } \\
\text { disorders: A } \\
\text { longitudinal } \\
\text { observation of pre } \\
\text { versus post } \\
\text { psychopathological } \\
\text { features in a sample of } \\
\text { patients with eating } \\
\text { disorders and a group } \\
\text { of healthy controls. }\end{array}$ & $\begin{array}{c}\text { Avaliar o impacto da } \\
\text { epidemia de COVID-19 em } \\
\text { pacientes com transtornos } \\
\text { alimentares considerando o } \\
\text { papel das vulnerabilidades } \\
\text { pré-existentes. }\end{array}$ & $\begin{array}{l}\text { Os resultados do estudo pontam que a epidemia } \\
\text { de COVID-19 teve impacto significativo sobre os } \\
\text { transtornos alimentares, tanto em termos de } \\
\text { sintomatologia pós-traumática quanto de } \\
\text { interferência no processo de recuperação. } \\
\text { Pacientes em remissão anterior mostraram } \\
\text { exacerbação de compulsão alimentar também } \\
\text { após o lockdown. Pacientes com bulimia nervosa } \\
\text { relataram sintomatologia pós-traumática } \\
\text { relacionada a COVID-19 mais grave do que } \\
\text { pacientes com anorexia nervosa e do grupo } \\
\text { controle. }\end{array}$ \\
\hline 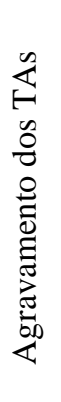 & $\begin{array}{l}\text { McCombie. } \\
\text { (2020) }\end{array}$ & $\begin{array}{l}\text { "Now It's Just Old } \\
\text { Habits and Misery"- } \\
\text { Understanding the } \\
\text { impact of the COVID- } \\
19 \text { pandemic on people } \\
\text { with current or life- } \\
\text { time eating disorders: a } \\
\text { qualitative Study. }\end{array}$ & $\begin{array}{l}\text { Compreender e identificar } \\
\text { os processos que estão na } \\
\text { base das respostas } \\
\text { psicológicas e } \\
\text { comportamentais das } \\
\text { pessoas com transtorno } \\
\text { alimentar durante a } \\
\text { pandemia de COVID-19. }\end{array}$ & $\begin{array}{l}\text { A amostra estudada indica uma piora ou } \\
\text { ressurgimento de quadros de transtornos } \\
\text { alimentares durante a pandemia. Isolamento, } \\
\text { ansiedade, falta de estrutura, interrupção de } \\
\text { rotinas e mensagens de mídia sobre peso e } \\
\text { exercícios pareciam contribuir para tal } \\
\text { agravamento. Sinaliza-se que a identificação de } \\
\text { tais aspectos se faz importante para que os } \\
\text { serviços possam se preparar para o acolhimento } \\
\text { de novos e antigos casos de transtornos } \\
\text { alimentares. }\end{array}$ \\
\hline 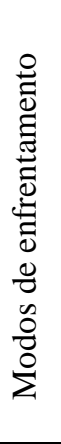 & $\begin{array}{l}\text { Schlegl et al. } \\
\quad(2020)\end{array}$ & $\begin{array}{l}\text { Eating disorders in } \\
\text { times of the COVID- } \\
19 \text { pandemic-Results } \\
\text { from an online survey } \\
\text { of patients with } \\
\text { anorexia nervosa. }\end{array}$ & $\begin{array}{c}\text { Explorar os efeitos da } \\
\text { pandemia de COVID-19 } \\
\text { nos transtornos alimentares } \\
\text { e outras consequências } \\
\text { psicológicas em pacientes } \\
\text { com anorexia nervosa que } \\
\text { passaram por internações } \\
\text { anteriores em uma clínica } \\
\text { na Alemanha. }\end{array}$ & $\begin{array}{l}\text { O estudo sugere que durante a pandemia de } \\
\text { COVID-19, os pacientes com anorexia nervosa } \\
\text { sofreram risco de agravamento dos transtornos } \\
\text { alimentares e sintomas gerais. Identificou-se } \\
\text { diminuição do acesso a psicoterapia presencial e } \\
\text { visitas ao clínico geral. Como estratégias de } \\
\text { enfrentamento, o estabelecimento de rotinas } \\
\text { diárias, o planejamento do dia e atividades } \\
\text { agradáveis foram consideradas as mais úteis. } \\
\text { Sinaliza-se que modalidades de tratamento mais } \\
\text { flexíveis devem ser consideradas no } \\
\text { acompanhamento e prevenção de recaídas. }\end{array}$ \\
\hline 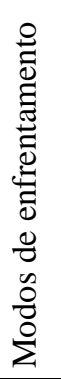 & $\begin{array}{l}\text { Richardson et } \\
\text { al. (2020) }\end{array}$ & $\begin{array}{l}\text { The impact of the } \\
\text { COVID-19 pandemic } \\
\text { on help-seeking } \\
\text { behaviors in } \\
\text { individuals suffering } \\
\text { from eating disorders } \\
\text { and their caregivers. }\end{array}$ & $\begin{array}{l}\text { Descrever o impacto da } \\
\text { pandemia de COVID-19 } \\
\text { nos comportamentos de } \\
\text { busca por ajuda entre os } \\
\text { indivíduos com transtornos } \\
\text { alimentares e cuidadores e } \\
\text { comparar com os anos de } \\
\text { 2018 e } 2019 .\end{array}$ & $\begin{array}{l}\text { A proporção de indivíduos afetados que } \\
\text { relataram comportamentos e/ou sintomas como } \\
\text { fazer dieta restritiva, excesso de exercício, } \\
\text { perfeccionismo, ansiedade e depressão foi maior } \\
\text { em } 2020 \text { em comparação com os anos anteriores. } \\
\text { Os achados apontam para o impacto da pandemia } \\
\text { de COVID-19 no agravamento dos sintomas de } \\
\text { transtornos alimentares e implicações negativas } \\
\text { para o acesso a serviços em tempos de crise. }\end{array}$ \\
\hline 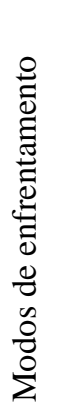 & $\begin{array}{l}\text { Monteleone et } \\
\text { al. (2021) }\end{array}$ & $\begin{array}{l}\text { Risk and resilience } \\
\text { factors for specific and } \\
\text { general } \\
\text { psychopathology } \\
\text { worsening in people } \\
\text { with Eating Disorders } \\
\text { during COVID-19 } \\
\text { pandemic: a } \\
\text { retrospective Italian } \\
\text { multicentre study. }\end{array}$ & $\begin{array}{c}\text { Avaliar quais fatores } \\
\text { contribuíram para a piora } \\
\text { induzida pelas restrições } \\
\text { impostas pela pandemia de } \\
\text { COVID-19 na } \\
\text { psicopatologia geral e } \\
\text { específica de pessoas com } \\
\text { transtornos alimentares. }\end{array}$ & $\begin{array}{c}\text { O estudo aponta que o medo do contágio, o } \\
\text { isolamento social e a baixa qualidade percebida } \\
\text { das relações terapêuticas são fatores de risco para } \\
\text { o agravamento da psicopatologia geral e } \\
\text { específica dos transtornos alimentares durante o } \\
\text { período de bloqueio pela COVID-19. A avaliação } \\
\text { e o destaque desses fatores de risco e resiliência } \\
\text { em potencial possivelmente contribuem para } \\
\text { melhor tratamento, bem como para se pensar } \\
\text { estratégias de prevenção para transtornos } \\
\text { alimentares. }\end{array}$ \\
\hline
\end{tabular}




\begin{tabular}{|c|c|c|c|c|}
\hline 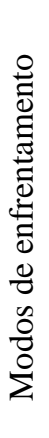 & $\begin{array}{l}\text { Baenas et al. } \\
\qquad(2020)\end{array}$ & $\begin{array}{l}\text { COVID-19 and eating } \\
\text { disorders during } \\
\text { confinement: Analysis } \\
\text { of factors associated } \\
\text { with resilience and } \\
\text { aggravation of } \\
\text { symptoms. }\end{array}$ & $\begin{array}{c}\text { Avaliar o nível de piora do } \\
\text { quadro clínico de pacientes } \\
\text { com transtornos } \\
\text { alimentares durante o } \\
\text { confinamento devido à } \\
\text { COVID-19 e examinar os } \\
\text { possíveis fatores que } \\
\text { prejudicam a adaptação a } \\
\text { esse contexto. }\end{array}$ & $\begin{array}{l}\text { O agravamento dos sintomas de transtornos } \\
\text { alimentares e psicopatologia geral (ansiedade e } \\
\text { depressão), durante o lockdown foi associado a } \\
\text { baixos escores na dimensão de caráter } \\
\text { relacionada ao autodirecionamento (TCR-I). O } \\
\text { aumento da sintomatologia de transtornos } \\
\text { alimentares durante o confinamento foi associado } \\
\text { a estratégias de enfrentamento menos adaptativas } \\
\text { para lidar com a situação de lockdown, tais como, } \\
\text { o excesso de informação sobre a COVID-19 ou a } \\
\text { presença de comportamentos alimentares } \\
\text { desordenados. }\end{array}$ \\
\hline 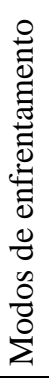 & $\begin{array}{l}\text { Clark Bryan et } \\
\text { al. }(2020)\end{array}$ & $\begin{array}{l}\text { Exploring the ways in } \\
\text { which COVID-19 and } \\
\text { lockdown has affected } \\
\text { the lives of adult } \\
\text { patients with anorexia } \\
\text { nervosa and their } \\
\text { carers. }\end{array}$ & $\begin{array}{c}\text { Explorar como a pandemia } \\
\text { de COVID-19 e as medidas } \\
\text { de bloqueio afetaram a } \\
\text { vida de pacientes adultos } \\
\text { com anorexia nervosa e de } \\
\text { seus cuidadores. }\end{array}$ & $\begin{array}{l}\text { O estudo identificou que o acesso reduzido aos } \\
\text { serviços de saúde, perda de rotina e } \\
\text { intensificação da ansiedade e sintomas de } \\
\text { transtornos alimentares representaram desafios } \\
\text { para pacientes e cuidadores. Maior suporte } \\
\text { remoto oferecido pelos serviços possibilitou a } \\
\text { continuidade do tratamento e o desenvolvimento } \\
\text { de recursos e estratégias de autogestão } \\
\text { promoveram autoeficácia em ambos os grupos. }\end{array}$ \\
\hline 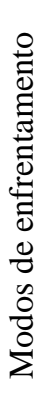 & $\begin{array}{l}\text { Graell et al. } \\
\text { (2020) }\end{array}$ & $\begin{array}{c}\text { Children and } \\
\text { adolescents with eating } \\
\text { disorders during } \\
\text { COVID-19 } \\
\text { confinement: } \\
\text { Difficulties and future } \\
\text { challenges. }\end{array}$ & $\begin{array}{l}\text { Apresentar as adaptações } \\
\text { dos protocolos de } \\
\text { tratamento desenvolvidos } \\
\text { em uma unidade de } \\
\text { atendimento a transtornos } \\
\text { alimentares em crianças e } \\
\text { adolescentes durante um } \\
\text { período de confinamento } \\
\text { de oito semanas. }\end{array}$ & $\begin{array}{l}\text { O estabelecimento rápido de um programa } \\
\text { combinado de teleterapia permitiu a continuidade } \\
\text { do tratamento ambulatorial e parcial do hospital- } \\
\text { dia, gerenciando a reativação de sintomas } \\
\text { alimentares e outras psicopatologias produzidas } \\
\text { durante o período. O tratamento de adolescentes } \\
\text { que requerem cuidados de hospital-dia foi } \\
\text { entendido como desafiador durante o } \\
\text { confinamento por apresentar maior gravidade e } \\
\text { instabilidade, exigindo maior número de } \\
\text { consultas e risco de internação. }\end{array}$ \\
\hline 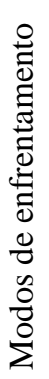 & $\begin{array}{l}\text { Fernández- } \\
\text { Aranda et } \\
\text { al.(2020) }\end{array}$ & $\begin{array}{l}\text { COVID Isolation } \\
\text { Eating Scale (CIES): } \\
\text { Analysis of the impact } \\
\text { of confinement in } \\
\text { eating disorders and } \\
\text { obesity-A } \\
\text { collaborative } \\
\text { international study. }\end{array}$ & $\begin{array}{l}\text { Analisar as propriedades } \\
\text { psicométricas da COVID } \\
\text { Isolation Eating Scale } \\
\text { (CIES); explorar as } \\
\text { alterações ocorridas devido } \\
\text { ao confinamento na } \\
\text { sintomatologia alimentar; e } \\
\text { explorar a aceitação geral } \\
\text { do uso de telemedicina } \\
\text { durante o confinamento. }\end{array}$ & $\begin{array}{l}\text { A adequação da CIES foi obtida pela } \\
\text { "confirmatory factor analysis," e os valores de } \\
\text { alfa de Cronbach variaram de bom a excelente. } \\
\text { Foi identificado que impactos positivos e } \\
\text { negativos do confinamento dependem do subtipo } \\
\text { de transtorno alimentar. Pacientes com anorexia } \\
\text { nervosa expressaram maior insatisfação e } \\
\text { dificuldade de acomodação com a terapia remota. }\end{array}$ \\
\hline
\end{tabular}

Fonte: Autores (2021).

\section{Discussão}

Para definir quais foram os impactos da pandemia de COVID-19 no desenvolvimento e manutenção de quadros de transtornos alimentares, identificou-se o uso muito variado de métodos e estratégias adotadas tanto a nível dos serviços de saúde quanto no nível individual. Em sua grande maioria os estudos utilizaram questionários online com perguntas adaptadas de escalas e testes já consagrados nas avaliações de transtornos alimentares e outros instrumentos de avaliação em saúde mental (Baenas et al., 2020; Branley-Bell \& Talbot, 2020; McCombie et al., 2020; Monteleone et al., 2021), mas também foram encontrados estudos qualitativos que buscaram realizar entrevistas semiestruturadas ou em profundidade (Brown et al., 2021; Clark Bryan et al., 2020; Termorshuizen et al., 2020) ou que associaram ambos os métodos (Machado et al., 2020). O recurso adotado para acessar a população estudada também variou bastante, uma vez que os informantes foram captados tanto em redes sociais (Branley-Bell \& Talbot, 2020; Brown et al., 2021; McCombie et al., 2020; Phillipou et al., 2020; Termorshuizen et al., 2020), quanto a partir do registro em clínicas e centros de referência para o tratamento de transtornos 
alimentares (Baenas et al., 2020; Castellini et al., 2020; Clark Bryan et al., 2020; Fernández-Aranda et al., 2020; Graell et al., 2020; Machado et al., 2020; Monteleone et al., 2021; Richardson et al., 2020; Schlegl et al., 2020).

Estima-se que entre um terço e metade da população exposta a uma pandemia possa vir a sofrer alguma manifestação psicopatológica, caso não seja feita nenhuma intervenção de cuidado específico para as reações emocionais e sintomas manifestados (Fundação Oswaldo Cruz., 2020). E, passados alguns meses desde o início da pandemia do COVID-19, estudos já apontavam para o aumento de depressão, estresse e ansiedade (Cao et al., 2020; Filgueiras \& Stults-Kolehmainen, 2020; Nguyen et al., 2020; Qiu et al., 2020; Vindegaard \& Benros, 2020).

Um recorte observado se relaciona a estudos populacionais que refletem a realidade de determinados países, onde o lockdown foi primeiramente adotado como medida de isolamento social. Nesses estudos, o impacto da pandemia em indivíduos com transtornos alimentares ou experiências de transtornos alimentares refletiu diretamente na diminuição da sensação de bem-estar psicológico. Tais sensações foram traduzidas pelos indivíduos pesquisados em redução da sensação de controle, aumento da sensação de isolamento social, aumento da reflexão sobre alimentação de forma desordenada, redução do sentimento de apoio social (Branley-Bell \& Talbot, 2020; Brown et al., 2021; Phillipou et al., 2020) e medo de ganhar peso e do que poderia acontecer caso contraíssem COVID-19 (Termorshuizen et al., 2020).

Esses efeitos psicológicos foram relacionados a uma desorganização dos comportamentos alimentares levando muitas vezes ao aumento da restrição alimentar, dos episódios de compulsão alimentar e dos comportamentos compensatórios (Brown et al., 2021; Phillipou et al., 2020; Termorshuizen et al., 2020). Apesar desse impacto negativo, os estudos também apontaram que o maior tempo em casa trouxe efeitos positivos refletindo, principalmente, na melhora no vínculo com a família e amigos, mais tempo para cuidar de si e de sua alimentação (Brown et al., 2021; Termorshuizen et al., 2020). Alguns informantes relataram até mesmo uma maior motivação para o tratamento e sensação de realização por conseguir lidar com o transtorno alimentar sem suporte profissional (Brown et al., 2021). Num dos estudos populacionais em que se estabeleceu comparação dos indivíduos com transtornos alimentares com a população em geral, um pequeno grupo relatou diminuição da restrição e compulsão alimentar durante a pandemia entre a população geral (Phillipou et al., 2020).

Os resultados apresentados corroboram os estudos que apontam o impacto desproporcional na saúde mental de alguns grupos (Pfefferbaum \& North, 2020; Reger et al., 2020), haja vista que fatores situacionais associados à pandemia de COVID19 podem influenciar de maneira significativa na precipitação dos transtornos alimentares, assim como na sintomatologia de quadros já instalados. Além disso, podem exacerbar as barreiras de acesso aos cuidados em saúde.

Dentre tais fatores, a necessidade de distanciamento físico pode privar os indivíduos do apoio social e ainda contribuir com possibilidade de se experimentar o isolamento social e a solidão, fatores de risco para problemas psicológicos em geral (Holt-Lunstad et al., 2015), bem como para desenvolvimento e manutenção de transtornos alimentares (Levine, 2012; Watson et al., 2017).

Cooper et al (2020) assinalam que o impacto do isolamento pode ser ainda mais grave para indivíduos com transtornos alimentares que também são membros de populações minoritárias, como por exemplo membros da comunidade LGBTQIA+, já que para esta população o apoio social é um componente crítico do funcionamento psicológico positivo. Além disso, as restrições à realização de exercícios físicos em academias e, em alguns momentos, de atividades físicas ao ar livre, podem levar a um aumento nas preocupações com o peso e forma corporal e impactar os padrões de alimentação, além de poderem contribuir para que os indivíduos se engajem em outros comportamentos compensatórios não saudáveis, como aumento da restrição alimentar ou métodos de purgação.

A maior parte dos estudos analisados se dedicou a avaliar o impacto dos fatores situacionais associados à pandemia, ainda que haja diferenças relevantes não só em relação aos métodos adotados, mas também, em relação ao tamanho da amostra e estratégia de análise de dados. Todos os estudos confirmaram que o lockdown impactou os indivíduos com transtornos 
alimentares, levando a um agravamento dos mais variados sintomas psicopatológicos dentre os quais destacamos aqueles relacionados à impulsividade, às dificuldades em lidar com as emoções positivas ou negativas, ao aumento da compulsão alimentar e da prática de exercícios físicos como forma de compensar a ingestão excessiva de alimentos, ao aumento da restrição alimentar, mau humor, ansiedade, sintomas afetivos, autoagressão e risco de suicídio (Castellini et al., 2020; Graell et al., 2020; Machado et al., 2020; McCombie et al., 2020).

Da mesma forma, a pandemia e as restrições sociais que a acompanham podem levar à redução da disponibilidade e do acesso aos alimentos, bem como à realização de estoques de alimentos em domicílio, o que pode representar per se um fator de risco para psicopatologia de transtornos alimentares, uma vez que reforça a predisposição a compulsão alimentar, jejum e comportamentos compensatórios (Becker et al., 2017; Lydecker \& Grilo, 2019; Rasmusson et al., 2019).

Ainda, o medo do contágio e a veiculação de mensagens de saúde pública que encorajam o aumento da atenção aos sintomas físicos potenciais de COVID-19 (como falta de ar, alterações no olfato e paladar, dores musculares, entre outros), podem levar os indivíduos a experimentarem uma hiper vigilância em relação ao próprio corpo, o que pode impactar em quadros de distorção de imagem corporal e sintomas alimentares. Somado a isto, o aumento na utilização de redes sociais observado desde o início da pandemia muito possivelmente pela escassez de interações sociais pessoais (Koeze \& Popper, 2020), vem sendo associado ao desenvolvimento ou agravamento de comportamentos alimentares tidos como desordenados e atitudes negativas em relação ao próprio corpo (Karsay et al., 2018; Sabik et al., 2020; Slater et al., 2017).

Outra categoria de estudos analisados especifica os modos de enfrentamento adotados pelos indivíduos durante a pandemia e pelos serviços de saúde que atendem pessoas com transtornos alimentares no sentido de minimizar os impactos prejudiciais da mesma. Todos os estudos relacionaram a pandemia de COVID-19 ao aumento do risco para o agravamento da psicopatologia geral e específica dos transtornos alimentares devido à utilização de estratégias de proteção menos eficazes com o contexto da pandemia (Baenas et al., 2020; Monteleone et al., 2021; Richardson et al., 2020; Schlegl et al., 2020). Isso implicou em adaptações no oferecimento dos serviços, haja vista a diminuição ao acesso ao tratamento presencial. Entre as estratégias de enfrentamento que se mostraram efetivas na proteção desses indivíduos em relação ao agravamento dos sintomas relacionados aos transtornos alimentares destacam-se, o estabelecimento de rotinas diárias, planejamento do dia e atividades agradáveis (Schlegl et al., 2020).

Vale considerar ainda que a forma de avaliar as estratégias adotadas pelos indivíduos e serviços de saúde é muito variada e pouco sistematizada trazendo dificuldades para a comparação entre os estudos. Um dos estudos (Baenas et al., 2020), por exemplo, citou no resumo do artigo e nos highlights um aumento de peso associado à utilização de estratégias de enfrentamento menos adaptativas, mas tal informação não está presente nos resultados do artigo. Observa-se que o que pode ser considerado como estratégia adaptativa ou menos adaptativa foi uniformizado, sem se levar em conta os possíveis efeitos variáveis destas estratégias entre os diferentes sujeitos de acordo com o tipo de transtorno alimentar.

Um dos estudos que buscaram definir protocolos de avaliação e tratamento para os transtornos alimentares associados ao tratamento remoto investiu na avaliação de adequação da COVID Isolation Eating Scale para avaliar a aceitação por parte dos pacientes dos recursos de telemedicina (Fernández-Aranda et al., 2020). Tendo em vista os diferentes subtipos de transtorno alimentar, o estudo identificou que pacientes com anorexia nervosa expressaram maior insatisfação e dificuldade de adaptação à terapia remota quando comparada com a terapia face a face. Além disso, identificou que pacientes com anorexia nervosa e obesidade obtiveram uma resposta positiva ao tratamento durante o confinamento, mas nenhuma mudança significativa foi encontrada em pacientes com bulimia nervosa. Pacientes com transtorno alimentar não especificado tiveram um aumento na sintomatologia alimentar e na psicopatologia (Fernández-Aranda et al., 2020).

Como estratégia de enfrentamento, os estudos identificaram que o impacto do COVID-19 em indivíduos com transtornos alimentares desafiam serviços e profissionais de saúde, forçando-os a construir novas formas de intervenção que 
respeitassem o distanciamento social. Dentre os elementos apontados pelos serviços de saúde, destaca-se a importância d e identificar como a situação de confinamento traz fatores relacionados à vulnerabilidade que são mais específicos entre os pacientes com transtornos alimentares do que a população em geral. A identificação desses fatores específicos se mostrou fundamental para a prevenção do agravamento dos sintomas relacionados aos transtornos alimentares, assim como na construção de abordagens personalizadas pata o tratamento desses indivíduos.

Assim, parte dos estudos mostraram que o atendimento remoto permitiu em muitos casos a continuidade do tratamento (Clark Bryan et al., 2020; Graell et al., 2020; Termorshuizen et al., 2020), ainda que tenha sido adotado por uma pequena parte dos indivíduos pesquisados (Schlegl et al., 2020). Embora a utilização de ferramentas de telessaúde representem uma alternativa, há barreiras para sua utilização e adesão. Nota-se que o uso dessas tecnologias para o atendimento de indivíduos com transtornos alimentares pode levar a mudanças nos modelos de cuidado e assistência, sendo necessário o desenvolvimento de estratégias que se desdobrem em métodos mais diversificados a fim de proporcionar um vínculo efetivo, corroborando para um acompanhamento individualizado (Fernández-Aranda et al., 2020).

\section{Conclusão}

Os estudos analisados, ainda que representem uma pequena amostra das pesquisas que já estão sendo publicadas em todo o mundo ao longo do ano de 2021, trazem importantes contribuições para pensarmos o impacto da pandemia de COVID19 em indivíduos com transtornos alimentares no Brasil, tendo em vista não só as especificidades do manejo da pandemia no país, mas também o fato de que não temos estudos populacionais sobre os transtornos alimentares em andamento.

As informações aqui apresentadas nos orientam em relação à construção de caminhos possíveis para sustentar o tratamento desses indivíduos durante o tempo prolongado de pandemia. Em um país de dimensões continentais como é o Brasil, as orientações do governo federal em relação à pandemia fragmentaram as estratégias em decisões regionais quase aleatórias, uma vez que divergiram das recomendações da Organização Mundial da Saúde. Por conseguinte, a sensação de insegurança e incerteza proliferaram de forma exponencial, causando considerável mal-estar na população geral. Trata-se ainda de avaliar em algum momento o impacto da inacreditável quantidade de pessoas afetadas direta e indiretamente em face à inabilidade do executivo federal em coordenar investimentos e ações para mitigar os efeitos devastadores da pandemia que até junho de 2021 contabiliza mais de 500 mil mortes.

As descobertas apresentadas associam inequivocamente os estágios iniciais da pandemia à exacerbação dos sintomas de transtornos alimentares, considerando as medidas de isolamento social e lockdown adotadas pela grande maioria dos países à exceção dos Estados Unidos e do Brasil que assumiram uma posição negacionista em relação à COVID-19 (Birman, 2020). Se essas medidas tiveram impacto significativo na saúde mental da população em geral por privá-la da vida cotidiana, não podemos negar que a escolha entre cumprir o isolamento social (e muitas vezes passar fome por não trabalhar) ou contrair a doença (por trabalhar) faz a presença constante da morte ser um fator de estresse, ansiedade e medo que não podem ser desconsiderados na especificidade brasileira.

Sendo assim, torna-se essencial a realização de estudos populacionais que permitam um melhor conhecimento da realidade dos transtornos alimentares no Brasil, assim como estudos futuros que avaliem as relações potenciais entre o contexto de pandemia e o desenvolvimento ou agravamento dos quadros de transtornos alimentares no país. Podemos considerar que tamanha distância entre a realidade brasileira - que mesmo antes da pandemia não realizava estudos populacionais relacionados aos transtornos alimentares - e a realidade dos países que desenvolveram esses estudos durante a realização efetiva de lockdown, nos mostra o tamanho do desafio que estamos enfrentando para levar a cabo os tratamentos que associam a gravidade dos transtornos alimentares a uma realidade que leva cotidianamente à deterioração da saúde mental dos 
brasileiros. Por outro lado, algumas estratégias adotadas e avaliadas pelos estudos analisados nos mostram o quanto é possível, pertinente e necessário ousar e criar em momentos de crise, ainda que a crise se arraste por longos anos.

\section{Agradecimentos}

Agradecemos à Fundação de Amparo à pesquisa do Rio de Janeiro (FAPERJ), à Coordenação de Aperfeiçoamento de Pessoal de Nível Superior (CAPES) e ao Conselho Nacional de Desenvolvimento Científico e Tecnológico (CNPq) pelo financiamento da pesquisa.

\section{Referências}

American Psychiatric Association. (2013). Diagnostic and Statistical Manual of Mental Disorders DSM-5 Fifth Edition (Editorial Reviews (ed.)).

Baenas, I., Caravaca-Sanz, E., Granero, R., Sánchez, I., Riesco, N., Testa, G., Vintró-Alcaraz, C., Treasure, J., Jiménez-Murcia, S., \& Fernández-Aranda, F. (2020). COVID-19 and eating disorders during confinement: Analysis of factors associated with resilience and aggravation of symptoms. European Eating Disorders Review, 28(6), 855-863. https://doi.org/10.1002/erv.2771

Becker, C. B., Middlemass, K., Taylor, B., Johnson, C., \& Gomez, F. (2017). Food insecurity and eating disorder pathology. International Journal of Eating Disorders, 50(9), 1031-1040. https://doi.org/10.1002/eat.22735

Birman, J. (2020). O trauma na pandemia do coronavírus: suas dimensões políticas, sociais, econômicas, ecológicas, culturais, éticas e científicas. (C. Brasileira (ed.).

Bourouiba, L. (2020). Turbulent Gas Clouds and Respiratory Pathogen Emissions: Potential Implications for Reducing Transmission of COVID-19. JAMA Journal of the American Medical Association, 323(18), 1837-1838. https://doi.org/10.1001/jama.2020.4756

Branley-Bell, D., \& Talbot, C. V. (2020). Exploring the impact of the COVID-19 pandemic and UK lockdown on individuals with experience of eating disorders. Journal of Eating Disorders, 8(1), 1-12. https://doi.org/10.1186/s40337-020-00319-y

Brown, S. M., Opitz, M. C., Peebles, A. I., Sharpe, H., Duffy, F., \& Newman, E. (2021). A qualitative exploration of the impact of COVID-19 on individuals with eating disorders in the UK. Appetite, 156. https://doi.org/10.1016/j.appet.2020.104977

Cao, W., Fang, Z., Hou, G., Han, M., Xu, X., Dong, J., \& Zheng, J. (2020). The psychological impact of the COVID-19 epidemic on college students in China. Psychiatry Research, 287. https://doi.org/10.1016/j.psychres.2020.112934

Castellini, G., Cassioli, E., Rossi, E., Innocenti, M., Gironi, V., Sanfilippo, G., Felciai, F., Monteleone, A. M., \& Ricca, V. (2020). The impact of COVID-19 epidemic on eating disorders: A longitudinal observation of pre versus post psychopathological features in a sample of patients with eating disorders and a group of healthy controls. International Journal of Eating Disorders, 53(11), 1855-1862. https://doi.org/10.1002/eat.23368

Clark Bryan, D., Macdonald, P., Ambwani, S., Cardi, V., Rowlands, K., Willmott, D., \& Treasure, J. (2020). Exploring the ways in which COVID-19 and lockdown has affected the lives of adult patients with anorexia nervosa and their carers. European Eating Disorders Review, 28(6), 826-835. https://doi.org/10.1002/erv.2762

Cooper, M., Reilly, E. E., Siegel, J. A., Coniglio, K., Sadeh-Sharvit, S., Pisetsky, E. M., \& Anderson, L. M. (2020). Eating disorders during the COVID-19 pandemic and quarantine: an overview of risks and recommendations for treatment and early intervention. Eating Disorders, 1-23. https://doi.org/10.1080/10640266.2020.1790271

Fernández-Aranda, F., Munguía, L., Mestre-Bach, G., Steward, T., Etxandi, M., Baenas, I., Granero, R., Sánchez, I., Ortega, E., Andreu, A., Moize, V. L., Fernández-Real, J. M., Tinahones, F. J., Diegüez, C., Frühbeck, G., Le Grange, D., Tchanturia, K., Karwautz, A., Zeiler, M., \& Jiménez-Murcia, S. (2020). COVID Isolation Eating Scale (CIES): Analysis of the impact of confinement in eating disorders and obesity-A collaborative international study. European Eating Disorders Review, 28(6), 871-883. https://doi.org/10.1002/erv.2784

Fernández-Aranda, F., Casas, M., Claes, L., Bryan, D. C., Favaro, A., Granero, R., Gudiol, C., Jiménez-Murcia, S., Karwautz, A., Le Grange, D., Menchón, J. M., Tchanturia, K., \& Treasure, J. (2020). COVID-19 and implications for eating disorders. European Eating Disorders Review, 28(3), 239-245. https://doi.org/10.1002/erv.2738

Filgueiras, A., \& Stults-Kolehmainen, M. (2020). The Relationship Between Behavioural and Psychosocial Factors Among Brazilians in Quarantine Due to COVID-19. SSRN Electronic Journal. https://doi.org/10.2139/ssrn.3566245

Fiorillo, A., \& Gorwood, P. (2020). The consequences of the COVID-19 pandemic on mental health and implications for clinical practice. European Psychiatry, 63(1). https://doi.org/10.1192/j.eurpsy.2020.35

Fundação Oswaldo Cruz. (2020). Saúde Mental e Atenção psicossocial na pandemia COVID-19 - Recomendações para gestores. Fiocruz/CEPEDES.

Graell, M., Morón-Nozaleda, M. G., Camarneiro, R., Villaseñor, Á., Yáñez, S., Muñoz, R., Martínez-Núñez, B., Miguélez-Fernández, C., Muñoz, M., \& Faya, M. (2020). Children and adolescents with eating disorders during COVID-19 confinement: Difficulties and future challenges. European Eating Disorders Review, 28(6), 864-870. https://doi.org/10.1002/erv.2763 
Holt-Lunstad, J., Smith, T. B., Baker, M., Harris, T., \& Stephenson, D. (2015). Loneliness and Social Isolation as Risk Factors for Mortality: A Meta-Analytic Review. Perspectives on Psychological Science, 10(2), 227-237. https://doi.org/10.1177/1745691614568352

Karsay, K., Knoll, J., \& Matthes, J. (2018). Sexualizing Media Use and Self-Objectification: A Meta-Analysis. Psychology of Women Quarterly, 42(1), 9-28. https://doi.org/10.1177/0361684317743019

Koeze, E., \& Popper, N. (2020). The Virus Changed the Way We Internet - The New York Times. The New York Times.

Levine, M. P. (2012). Loneliness and eating disorders. Journal of Psychology: Interdisciplinary and Applied, 146(1-2), 243-257. https://doi.org/10.1080/00223980.2011.606435

Lydecker, J. A., \& Grilo, C. M. (2019). Food insecurity and bulimia nervosa in the United States. International Journal of Eating Disorders, 52(6), 735-739. https://doi.org/10.1002/eat.23074

Machado, P. P. P., Pinto-Bastos, A., Ramos, R., Rodrigues, T. F., Louro, E., Gonçalves, S., Brandão, I., \& Vaz, A. (2020). Impact of COVID-19 lockdown measures on a cohort of eating disorders patients. Journal of Eating Disorders, 8(1), 4-11. https://doi.org/10.1186/s40337-020-00340-1

McCombie, C., Austin, A., Dalton, B., Lawrence, V., \& Schmidt, U. (2020). "Now It's Just Old Habits and Misery"-Understanding the Impact of the Covid19 Pandemic on People With Current or Life-Time Eating Disorders: A Qualitative Study. Frontiers in Psychiatry, 11. https://doi.org/10.3389/fpsyt.2020.589225

Monteleone, A. M., Cascino, G., Marciello, F., Abbate-Daga, G., Baiano, M., Balestrieri, M., Barone, E., Bertelli, S., Carpiniello, B., Castellini, G., Corrivetti, G., De Giorgi, S., Favaro, A., Gramaglia, C., Marzola, E., Meneguzzo, P., Monaco, F., Oriani, M. G., Pinna, F., Monteleone, P. (2021). Risk and resilience factors for specific and general psychopathology worsening in people with Eating Disorders during COVID-19 pandemic: a retrospective Italian multicentre study. Eating and Weight Disorders, 1, 3. https://doi.org/10.1007/s40519-020-01097-x

Morawska, L., \& Cao, J. (2020). Airborne transmission of SARS-CoV-2: The world should face the reality. Environment International, 139. https://doi.org/10.1016/j.envint.2020.105730

Nguyen, H. C., Nguyen, M. H., Do, B. N., Tran, C. Q., Nguyen, T. T. P., Pham, K. M., Pham, L. V., Tran, K. V., Duong, T. T., Tran, T. V., Duong, T. H., Nguyen, T. T., Nguyen, Q. H., Hoang, T. M., Nguyen, K. T., Pham, T. T. M., Yang, S.-H., Chao, J. C.-J., \& Duong, T. Van. (2020). People with Suspected COVID-19 Symptoms Were More Likely Depressed and Had Lower Health-Related Quality of Life: The Potential Benefit of Health Literacy. Journal of Clinical Medicine, 9(4), 965. https://doi.org/10.3390/jcm9040965

Pfefferbaum, B., \& North, C. S. (2020). Mental Health and the Covid-19 Pandemic. New England Journal of Medicine, 383(6), 510-512. https://doi.org/10.1056/nejmp2008017

Phillipou, A., Meyer, D., Neill, E., Tan, E. J., Toh, W. L., Van Rheenen, T. E., \& Rossell, S. L. (2020). Eating and exercise behaviors in eating disorders and the general population during the COVID-19 pandemic in Australia: Initial results from the COLLATE project. International Journal of Eating Disorders, 53(7), 1158-1165. https://doi.org/10.1002/eat.23317

Qiu, J., Shen, B., Zhao, M., Wang, Z., Xie, B., \& Xu, Y. (2020). A nationwide survey of psychological distress among Chinese people in the COVID-19 epidemic: implications and policy recommendations. General Psychiatry, 33(2), e100213. https://doi.org/10.1136/gpsych-2020-100213

Rasmusson, G., Lydecker, J. A., Coffino, J. A., White, M. A., \& Grilo, C. M. (2019). Household food insecurity is associated with binge-eating disorder and obesity. International Journal of Eating Disorders, 52(1), 28-35. https://doi.org/10.1002/eat.22990

Reger, M. A., Stanley, I. H., \& Joiner, T. E. (2020). Suicide Mortality and Coronavirus Disease 2019-A Perfect Storm? JAMA Psychiatry, 77(11), 1093-1094. https://doi.org/10.1001/jamapsychiatry.2020.1060

Richardson, C., Patton, M., Phillips, S., \& Paslakis, G. (2020). The impact of the COVID-19 pandemic on help-seeking behaviors in individuals suffering from eating disorders and their caregivers. General Hospital Psychiatry, 67, 136-140. https://doi.org/10.1016/j.genhosppsych.2020.10.006

Sabik, N. J., Falat, J., \& Magagnos, J. (2020). When Self-Worth Depends on Social Media Feedback: Associations with Psychological Well-Being. Sex Roles, 82(7-8), 411-421. https://doi.org/10.1007/s11199-019-01062-8

Saunders, J. F., Eaton, A. A., \& Fitzsimmons-Craft, E. E. (2019). Body-, Eating-, and Exercise-Related Comparisons During Eating Disorder Recovery and Validation of the BEECOM-R. Psychology of Women Quarterly, 43(4), 494-508. https://doi.org/10.1177/0361684319851718

Schlegl, S., Maier, J., Meule, A., \& Voderholzer, U. (2020). Eating disorders in times of the COVID-19 pandemic-Results from an online survey of patients with anorexia nervosa. International Journal of Eating Disorders, 53(11), 1791-1800. https://doi.org/10.1002/eat.23374

Slater, A., Varsani, N., \& Diedrichs, P. C. (2017). \#fitspo or \#loveyourself? The impact of fitspiration and self-compassion Instagram images on women's body image, self-compassion, and mood. Body Image, 22, 87-96. https://doi.org/10.1016/j.bodyim.2017.06.004

Souza, M. T., Silva, M. D., \& Carvalho, R. (2010). Integrative review: what is it? How to do it? Einstein (São Paulo), 8(1), 102-106. https://doi.org/10.1590/s1679-45082010rw1134

Termorshuizen, J. D., Watson, H. J., Thornton, L. M., Borg, S., Flatt, R. E., MacDermod, C. M., Harper, L. E., van Furth, E. F., Peat, C. M., \& Bulik, C. M. (2020). Early impact of COVID-19 on individuals with self-reported eating disorders: A survey of $\sim 1,000$ individuals in the United States and the Netherlands. International Journal of Eating Disorders, 53(11), 1780-1790. https://doi.org/10.1002/eat.23353

Torales, J., O’Higgins, M., Castaldelli-Maia, J. M., \& Ventriglio, A. (2020). The outbreak of COVID-19 coronavirus and its impact on global mental health. International Journal of Social Psychiatry, 66(4), 317-320. https://doi.org/10.1177/0020764020915212 
Research, Society and Development, v. 10, n. 10, e418101019015, 2021

(CC BY 4.0) | ISSN 2525-3409 | DOI: http://dx.doi.org/10.33448/rsd-v10i10.19015

Vindegaard, N., \& Benros, M. E. (2020). COVID-19 pandemic and mental health consequences: Systematic review of the current evidence. Brain, Behavior, and Immunity, 89, 531-542. https://doi.org/10.1016/j.bbi.2020.05.048

Wang, C., Pan, R., Wan, X., Tan, Y., Xu, L., Ho, C. S., \& Ho, R. C. (2020). Immediate psychological responses and associated factors during the initial stage of the 2019 coronavirus disease (COVID-19) epidemic among the general population in China. International Journal of Environmental Research and Public Health, 17(5). https://doi.org/10.3390/ijerph17051729

Watson, R. J., Veale, J. F., \& Saewyc, E. M. (2017). Disordered eating behaviors among transgender youth: Probability profiles from risk and protective factors. International Journal of Eating Disorders, 50(5), 515-522. https://doi.org/10.1002/eat.22627

World Health Organization. (2020a). Considerations for quarantine of individuals in the context of containment for coronavirus disease (COVID-19). In Who (Issue February).

World Health Organization. (2020b). General's Opening Remarks at the Media Briefing on COVID-19-11 March 2020. World Health Organization. https://www.who.int/director-general/speeches/detail/who-director-general-s-opening-remarks-at-the-media-briefing-on-covid-19---20-march-2020

World Health Organization. (2020c). Responding to community spread of COVID-19 Interim guidance 7 March 2020 Background.

World Health Organization. (2020d). WHO Director-General's statement on IHR Emergency Committee on Novel Coronavirus. WHO Director-General $\mid$ Speeches. https://www.who.int/director-general/speeches/detail/who-director-general-s-statement-on-ihr-emergency-committee-on-novel-coronavirus

World Health Organization. (2021). WHO Coronavirus (COVID-19) Dashboard. WHO Coronavirus (COVID-19). https://covid19.who.int/ 